\title{
Hybrid Orthogonal Frequency Division Multiplexing with Subcarrier Number Modulation
}

\author{
Jun $\mathrm{Li}^{1}$, Shuping Dang ${ }^{2}$, Miaowen $\mathrm{Wen}^{3}$, Shahid Mumtaz ${ }^{4}$, Qiang $\mathrm{Li}^{5}$, and Constandinos X. Mavromoustakis ${ }^{6}$ \\ ${ }^{1}$ Research Center of Intelligent Communication Engineering, \\ School of Electronics and Communication Engineering, \\ Guangzhou University, Guangzhou 510006, China \\ ${ }^{2}$ Computer, Electrical and Mathematical Science and Engineering Division, \\ King Abdullah University of Science and Technology (KAUST), \\ Thuwal 23955-6900, Kingdom of Saudi Arabia \\ ${ }^{3}$ School of Electronic and Information Engineering, \\ South China University of Technology, Guangzhou 510640, China \\ ${ }^{4}$ Instituto de Telecomunicacoes, 112148 Lisboa, Portugal \\ ${ }^{5}$ College of Information Science and Technology, Jinan University, Guangzhou 510632, China \\ ${ }^{6}$ Department of Computer Science, University of Nicosia, CY-1700 Nicosia, Cyprus \\ Email: eemwwen@scut.edu.cn
}

\begin{abstract}
In this paper, we propose a hybrid OFDM-SNM scheme, named joint-mapping OFDM-SNM (JM-OFDM-SNM), to avoid transmitting variable lengths of information bits. In JM-OFDM-SNM, the signal vectors are generated by jointly considering subcarrier activation patterns and constellation symbols. To relieve the high computational complexity of the optimal maximum-likelihood (ML) detection, we design a lowcomplexity detection method via resorting to the log-likelihood ratio criterion. We also analyze the upper bound on the bit error rate of JM-OFDM-SNM. To further enhance the utilization of frequency resource, we propose a more general scheme, named adaptive JM-OFDM-SNM (AJM-OFDM-SNM), to accommodate the constellation orders for different numbers of activated subcarriers. Simulation results show that AJM-OFDM-SNM achieves better performance than both JM-OFDM-SNM and OFDM-SNM at the same spectral efficiency. The low-complexity detection method of JM-OFDM-SNM achieves very close performance to the optimal ML detection, and the theoretical curves well match the simulation curves in the high signal-to-noise ratio region.

Index Terms-Orthogonal frequency division multiplexing (OFDM), subcarrier number modulation, bit error rate (BER), low-complexity detection.
\end{abstract}

\section{INTRODUCTION}

Due to the rapid growth in the number of mobile users and smart devices, several novel techniques are proposed to meet the demands of high date rate, high spectral efficiency (SE), and high energy efficiency (EE) for the next sixth generation (6G) networks [1]-[3]. Index modulation (IM), as one of novel transmission techniques, is proposed to achieve the high SE and high EE [4]. In IM, the pattern index(es) are utilized as the extra information-carrying units, where the pattern refers to the activation states of time slots, subcarriers, transmit antennas, receive antennas, linear block codes, and etc [5].

Recently, the IM paradigm is applied to the frequency domain by utilizing the indices of orthogonal frequency division multiplexing (OFDM) subcarriers to transmit index bits. In [6], OFDM with IM (OFDM-IM) is proposed to transmit the indices of a group of subcarriers as well as the modulated symbols via the activated subcarriers. Similar to SM, information bits for OFDM-IM are also split into index bits and modulation bits, where index bits and modulation bits determine the subcarrier activation pattern (SAP) and the modulated symbols, respectively. Because of the special configuration, OFDM-IM obtains better performance than the conventional OFDM scheme. Attracted by its merits, lots of research has been proposed to exploit the potential of OFDM-IM, e.g., fidelity improvement and SE improvement. For instance, the interleaved subcarrier grouping method is proposed to improve the bit error rate (BER) performance of OFDM-IM in [7]. Enhanced OFDM-IM is proposed to enhance the BER performance of OFDM-IM by extending the signals to in-phase/quadrature domains in [8]. It should be noted that not all SAPs are used for transmission, which restricts the BER performance of OFDM-IM. Therefore, a special constellation design is proposed to improve the BER performance of OFDM-IM by activating all SAPs for transmission in [9]. To increase the SE of OFDM-IM, a generalized OFDM-IM scheme is proposed to achieve a large number of index bits in [10]. In [11], authors propose a layered OFDMIM scheme to increase the number of index bits by selecting SAPs via multiple layers. In addition, OFDM-IM is further applied to the conventional MIMO system to largely increase its SE [12].

It is worth noting that the all above OFDM-IM related schemes only activate partial subcarriers for transmission, which wastes the frequency resource so as to limit the improvement of SE. Therefore, in order to break this limit, dualmode IM aided OFDM (DM-IM-OFDM) is proposed to utilize both selected and unselected subcarriers to transmit modulated symbols [13]. In a similar manner, the authors in [14] also de- 
sign the multiple-mode OFDM-IM (MM-OFDM-IM) scheme to transmit modulated symbols via a full set of subcarriers. Note that DM-IM-OFDM maps index bits to the selected SAP (indices of selected subcarriers), while MM-OFDM-IM maps index bits to the permutation of distinguishable constellations. Except for the above research works, the low-complexity detection design is also an important research direction for OFDM-IM [15], [16]. However, although OFDM-IM has many advantages, disadvantages still exist. For example, OFDM-IM transmits its own signals through a fixed number of active subcarriers, which has not fully exploited the SE advantage of IM. Furthermore, the mapping method between index bits and SAP is very complicated, especially when the number of subcarriers goes large. Therefore, to mitigate these problems, OFDM with subcarrier number modulation (OFDM-SNM) is proposed to convey index bits via the number of subcarriers [17]-[19]. Note that the number of active subcarriers in OFDM-SNM is varied, which can thereby achieve a higher SE than OFDMIM under certain conditions. Nevertheless, OFDM-SNM has a serious drawback that the length of information bits is varied depending on the number of active subcarriers, which leads to the error propagation problem.

In order to solve this drawback of OFDM-SNM, we propose a joint-mapping OFDM-SNM (JM-OFDM-SNM) scheme to generate the fixed length of information bits for each transmission. In JM-OFDM-SNM, the possible transmitted vectors are generated by considering all possible SAPs and the corresponding modulated symbols. After obtaining all possible transmitted vectors, a bijective mapping is implemented based on the information bits. In addition, the lowcomplexity detection method is proposed to relieve the high detection load of maximum-likelihood (ML) detection, and the theoretical analysis is also investigated. Note that the number of transmitted vectors for JM-OFDM-SNM is not always a power of two, and partial transmitted vectors will not be used at all, which affects the transmission rate and BER performance of JM-OFDM-SNM. Therefore, to improve the BER performance and transmission rate of JM-OFDM-SNM, we further propose the adaptive JM-OFDM-SNM (AJMOFDM-SNM) scheme, which selects the modulated symbols from different constellations for different numbers of activated subcarriers. Simulation results show that (A)JM-OFDM-SNM obtains better performance than OFDM-SNM under the same transmission rate. As also shown by the numerical results, the proposed low-complexity detection scheme for JM-OFDMSNM approaches the optimal ML detection with the slight performance loss, and the theoretical curves well match the simulation curves.

Notations: Upper and lower case boldface letters denote matrices and column vectors, respectively. The complex number field is represented by $\mathbb{C}$. $(\cdot)^{T}$ and $(\cdot)^{H}$ represent the transpose and Hermitian transpose operations, respectively. $\mathbf{I}_{M}$ is an $M \times M$ identity matrix. $E\{\cdot\}$ denotes the expectation operation. $\|\cdot\|$ denotes the Frobenius norm operation of the argument. $C(\cdot, \cdot)$ represent the binomial operation of the argument. $Q(\cdot)$ denotes the Gaussian $Q$-function. $\operatorname{rank}\{\cdot\}$

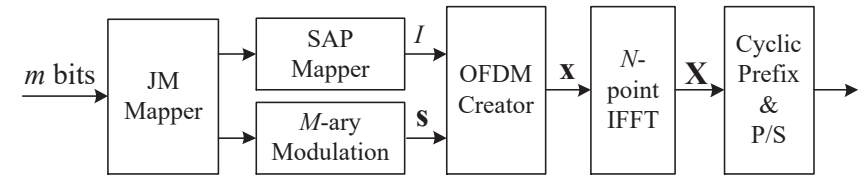

Fig. 1. System model of JM-OFDM-SNM.

TABLE I

MAPPING TABLE OF JM-OFDM-SNM WITH $N=4, V=3$ AND BPSK.

\begin{tabular}{c||c||c||c}
\hline $\mathrm{v}$ & $k$ & Input Bits & $\mathbf{x}_{k}$ \\
\hline \hline 1 & 1 & 000000 & {$[-1,0,0,0]^{T}$} \\
1 & 2 & 000001 & {$[+1,0,0,0]^{T}$} \\
$\vdots$ & $\vdots$ & $\vdots$ & $\vdots$ \\
2 & 31 & 011110 & {$[0,0,+1,-1]^{T}$} \\
2 & 32 & 011111 & {$[0,0,+1,+1]^{T}$} \\
$\vdots$ & $\vdots$ & $\vdots$ & $\vdots$ \\
3 & 63 & 111110 & {$[0,+1,+1,-1]^{T}$} \\
3 & 64 & 111111 & {$[0,+1,+1,+1]^{T}$} \\
\hline
\end{tabular}

indicates the rank of the argument. $\operatorname{diag}\{\mathbf{x}\}$ denotes a diagonal matrix whose diagonal elements are drawn from $\mathbf{x} \cdot \operatorname{det}\{\cdot\}$ represents the determinant of the argument. The probability of an event is denoted by $\operatorname{Pr}(\cdot) .\lfloor\cdot\rfloor$ indicates the floor operation.

\section{Proposed (A)JM-OFDM-SNM SchemE}

The system model of JM-OFDM-SNM is depicted in Fig. 1. In JM-OFDM-SNM, $V$ out of totally $N$ subcarriers are allowed to be activated with $1 \leq V \leq N$. Similar to OFDM-SNM, the number of activated subcarriers is varied. However, unlike the mapping strategy between only index bits and SAP in OFDM-SNM, JM-OFDM-SNM investigates a joint mapping rule between all input bits and the transmitted vectors. Actually, there are totally $N_{T}=\sum_{v=1}^{V} C(N, v)$ SAPs. For each SAP, $v$ subcarrier are active, and thus $v$ modulated symbols $\mathbf{S}=\left[s_{1}, \ldots, s_{v}\right]$ can be transmitted with the corresponding SAP $I=\left[i_{1}, \ldots, i_{v}\right]$. Considering all candidates for $v$ subcarriers and modulated symbols, the number of all possible transmitted vectors can be calculated as

$$
N_{A}=\sum_{v=1}^{V} C(N, v) M^{v} .
$$

Since the total number of transmitted vectors $N_{A}$ is fixed, the constant length of input bits ( $m$ bits) for JM-OFDM-SNM can be obtained by a bijective mapping scheme between input bits and $N_{A}$ transmitted vectors, which results in

$$
\bar{r}_{\mathrm{JM}}=m=\left\lfloor\log _{2}\left(N_{A}\right)\right\rfloor .
$$

Note that $N_{A}$ may not be a power of two, and thus only $F=2^{\left\lfloor\log _{2}\left(N_{A}\right)\right\rfloor}$ transmitted vectors are used for transmission.

The transmitted vector for JM-OFDM-SNM in the frequen- 
cy domain can be obtained by combing $I$ and $\mathbf{s}$ as

$$
\mathbf{x}_{k}=\left[0, s_{1}, 0, s_{2}, 0, \ldots, s_{v}\right]^{T},
$$

where $1 \leq k \leq F$.

We provide an example of JM-OFDM-SNM for illustration with $N=4, V=3$ and BPSK. In this case, there are totally $N_{A}=\sum_{v=1}^{3} C(4, v) 2^{v}=64$ possible transmitted vectors, which lead to totally 6 input bits $(m=6)$ and the corresponding mapping table is given in Table I. From Table I, the transmitted vector $\mathbf{x}_{k}$ can be directly obtained by certain input bits. For instance, input bits are assumed to be 011110 with $k=31$ and the corresponding transmitted vector is given by $\mathbf{x}_{31}=[0,0,+1,-1]^{T}$. If input bits are 000001 with $k=2$, the transmitted vector are then obtained by $\mathbf{x}_{2}=[+1,0,0,0]^{T}$.

Compared to the conventional OFDM-SNM, JM-OFDMSNM achieves a constant length of input bits, which avoids the error propagation effect. Moreover, not all subcarriers need to be activated for transmission by JM-OFDM-SNM, which optimizes the EE.

The following process is the same as the conventional OFDM scheme that $N$-point IFFT is implemented to $\mathbf{x}_{k}$ and the resulting time-domain signal is thereby given by $\mathbf{X}^{k}=\left[X_{1}^{k}, X_{2}^{k}, \ldots, X_{N}^{k}\right]^{T}$, which will be transmitted to the receiver after $\mathrm{CP}$ insertion and $\mathrm{P} / \mathrm{S}$ conversion.

By going through the fading channels, the frequencydomain received signal vector can be expressed by

$$
\mathbf{y}=\frac{1}{\sqrt{\bar{v}}} \mathbf{H} \mathbf{x}_{k}+\mathbf{n},
$$

where $\mathbf{H}=\operatorname{diag}\{\mathbf{h}\}=\operatorname{diag}\left\{h_{1}, h_{2}, \ldots, h_{N}\right\}$ denotes the channel coefficient matrix whose entries follow the complex Gaussian distribution with zero mean and unit variance; $\bar{v}$ denotes the average number of active subcarriers; $\mathbf{n}$ represents the additive Gaussian noise vector with zero mean and variance $N_{0}$. The transmitted signal can be estimated by employing the optimal ML detection by

$$
\hat{\mathbf{x}}_{k}=\underset{\dot{\mathbf{x}}_{k}}{\arg \min }\left\|\mathbf{y}-\frac{1}{\sqrt{v}} \mathbf{H} \dot{\mathbf{x}}_{k}\right\|^{2} .
$$

It can be seen from (5) that the optimal ML detection brings an exhaustive search over all possible candidates of the transmitted vector $\mathbf{x}_{k}$, which results in high computational complexity, especially for large values of $N, V$, or $M$.

From (1), we can see that the total number of all possible transmitted vectors $N_{A}$ in JM-OFDM-SNM is probably not a power of two, and $N_{A}-F$ transmitted vectors (redundancy) should be discarded for modulation purposes, which leads to a serious waste of subcarrier resources and limits the transmission rate. In the following, we then propose the AJMOFDM-SNM scheme to adaptively generate the modulated symbols from different constellations for different numbers of activated subcarriers $(v)$ so as to improve the transmission rate and BER of JM-OFDM-SNM. Specifically, in order to make full use of all $N_{A}$ transmitted vectors, we can adapt the cardinality of the constellation set for each $v$ so as to achieve a higher transmission rate or a lower BER, which results in a total number of transmitted vectors

$$
\bar{N}_{A}=\sum_{v=1}^{V} C(N, v)\left(M_{v}\right)^{v}
$$

where $M_{v}$ denotes the cardinality of the constellation set used for the SAP with $v$ active subcarriers. Note that JMOFDM-SNM can be considered as a special case of AJMOFDM-SNM with $M_{1}=M_{2}=\cdots=M_{V}$. Obviously, the transmission rate of AJM-OFDM-SNM is given by

$$
\bar{r}_{\mathrm{AJM}}=\bar{m}=\left\lfloor\log _{2}\left(\bar{N}_{A}\right)\right\rfloor .
$$

It can be seen that compared to JM-OFDM-SNM, AJMOFDM-SNM is able to not only achieve a higher transmission rate but also reduce the number of redundant transmitted vectors by properly adjusting the constellation order for different numbers of activated subcarriers. In other words, AJMOFDM-SNM is more flexible and practical than JM-OFDMSNM. To clearly exhibit the mapping rule between input bits and transmitted vectors, let us take AJM-OFDM-SNM with $N=4, V=2$, and [BPSK, QPSK] as an example, which has 64 legal transmitted vectors and a transmission rate of 1.5 bpcu. Assume the input bits are 00000 and the corresponding transmitted vector is given by $\overline{\mathbf{x}}_{1}=[-1,0,0,0]^{T}$. If the input bits are 11111 , the transmitted vector is then given by $\overline{\mathbf{x}}_{64}=\left[0,0, \frac{1+j}{\sqrt{2}}, \frac{1+j}{\sqrt{2}}\right]^{T}$. It is easy to find that modulated symbols in $\overline{\mathbf{x}}_{1}$ and $\overline{\mathbf{x}}_{6} 4$ are selected from BPSK and QPSK, respectively.

\section{LOW-COMPlexity Detection AND PeRformance ANALYSIS}

In this section, we propose a log-likelihood ratio (LLR) detection method, which largely reduces the computational complexity of the optimal ML detection. The detection metric can be formulated as

$$
L(\alpha)=\ln \frac{\operatorname{Pr}\left(\mathrm{A}_{\alpha} \mid y(\alpha)\right)}{\operatorname{Pr}\left(\overline{\mathrm{A}}_{\alpha} \mid y(\alpha)\right)}=\ln \frac{\operatorname{Pr}\left(\mathrm{A}_{\alpha}\right) f\left(y(\alpha) \mid \mathrm{A}_{\alpha}\right)}{\operatorname{Pr}\left(\overline{\mathrm{A}}_{\alpha}\right) f\left(y(\alpha) \mid \overline{\mathrm{A}}_{\alpha}\right)},
$$

where $y(\alpha)$ denotes the $\alpha$ th element of $\mathbf{y}, \mathbf{A}_{\alpha}$ denotes the event that the $\alpha$ th subcarrier is active with the probability $\operatorname{Pr}\left(\mathrm{A}_{\alpha}\right)=$ $\frac{\sum_{v=1}^{V} v C(N, v)}{N T}$ with $T=\sum_{v=1}^{V} C(N, v)$, and $\overline{\mathrm{A}}_{\alpha}^{v}$ represents the complementary event of $\mathrm{A}_{\alpha}^{v}$ with $\operatorname{Pr}\left(\overline{\mathrm{A}}_{\alpha}\right)=1-\operatorname{Pr}\left(\mathrm{A}_{\alpha}\right)$. The conditional PDF $f\left(y(\alpha) \mid \mathrm{A}_{\alpha}\right)$ is explicitly expressed in (9) at the top of the next page.

After calculating all values of $L(\alpha)$ with $1 \leq \alpha \leq$ $N$, we obtain the $\mathrm{LLR}$ vector $\mathbf{L}=[L(1), \ldots, L(N)]$ and the correspondingly estimated modulation symbols $\hat{\mathbf{s}}=$ $[\hat{s}(1), \ldots, \hat{s}(N)]$. It can be seen from (8) that the $\alpha$ th subcarrier is more likely to be activated with a larger value of $L(\alpha)$. In order to figure out the possible number and the corresponding indices of active subcarriers, we first sort the values of $\mathbf{L}$ as $\left[z_{1}, \ldots, z_{N}\right]=\operatorname{sort}([L(1), \ldots, L(N)])$, where sort $(\cdot)$ denotes the ordering function that reorders the elements in descending order, and $z_{1}\left(z_{N}\right)$ denotes the position of the maximal (minimal) value in $\mathbf{L}$. Therefore, the $V$ possible SAPs 


$$
f\left(y(\alpha) \mid \mathrm{A}_{\alpha}\right)= \begin{cases}\frac{1}{\pi \sigma^{2}} \exp \left(-\frac{1}{\sigma^{2}}\left|y(\alpha)-\frac{1}{\sqrt{v}} h_{\alpha} \hat{s}(\alpha)\right|^{2}\right), & \text { if the } \alpha \text { th subcarrier is active } \\ \frac{1}{\pi \sigma^{2}} \exp \left(-\frac{1}{\sigma^{2}}|y(\alpha)|^{2}\right), & \text { if the } \alpha \text { th subcarrier is inactive. }\end{cases}
$$

can be estimated as

$$
\hat{I}_{1}=\left[z_{1}\right], \hat{I}_{2}=\left[z_{1}, z_{2}\right], \ldots, \hat{I}_{V}=\left[z_{1}, \ldots, z_{V}\right],
$$

where $\hat{I}_{v}$ indicates the estimated SAP for activating $v$ subcarriers with $1 \leq v \leq V$. To find the final estimation of SAP (or the number of active subcarriers $v$ ), we need to further compare the Euclidean distances of all $V$ possible SAP as follows:

$$
\hat{v}=\underset{\left\{\hat{I}_{v}\right\}_{v=1}^{V}, \mathbf{s}}{\arg \min }\left\|\mathbf{y}-\frac{1}{\sqrt{\bar{v}}} \mathbf{H} \mathbf{x}_{v}^{\prime}\right\|^{2},
$$

where $\mathbf{x}_{v}^{\prime}=[0, \hat{s}(1), \ldots, \hat{s}(v)]$ with the non-zero elements in the positions of $\hat{I}_{v}$. After obtaining $\hat{v}$, the estimated signal vector $\hat{\mathbf{x}}$ can be directly constructed by $\hat{I}_{\hat{v}}$ and $\hat{\mathbf{s}}$. Finally, the information bits are extracted by demapping $\hat{\mathbf{x}}$.

We then theoretically deduce the upper bound on the BER of JM-OFDM-SNM assuming the perfect channel state information at the receiver. According to (5), the conditional pairwise error probability (PEP) on $\mathbf{H}$ is given by

$$
\begin{aligned}
\operatorname{Pr}\left\{\mathbf{x}_{k} \rightarrow \hat{\mathbf{x}}_{k} \mid \mathbf{H}\right\} & =\operatorname{Pr}\left\{\left\|\mathbf{y}-\frac{\mathbf{H} \mathbf{x}_{k} \|^{2}}{\sqrt{\bar{v}}}\right\|^{2}\left\|\mathbf{y}-\frac{\mathbf{H} \hat{\mathbf{x}}_{k}}{\sqrt{\bar{v}}}\right\|^{2}\right\} \\
& =Q\left(\sqrt{\frac{\left\|\mathbf{H}\left(\mathbf{x}_{k}^{\prime}-\hat{\mathbf{x}}_{k}^{\prime}\right)\right\|^{2}}{2 N_{0}}}\right),
\end{aligned}
$$

where $\mathbf{x}_{k}^{\prime}=\mathbf{x}_{k} / \sqrt{\bar{v}}$ and $\hat{\mathbf{x}}_{k}^{\prime}=\hat{\mathbf{x}}_{k} / \sqrt{\bar{v}}$. Applying the $Q$ function approximation [20]

$$
Q(x) \cong \frac{1}{12} e^{-\frac{x^{2}}{2}}+\frac{1}{4} e^{-\frac{2 x^{2}}{3}},
$$

the unconditional PEP can be approximated as

$$
\begin{gathered}
\operatorname{Pr}\left\{\mathbf{x}_{k} \rightarrow \hat{\mathbf{x}}_{k}\right\}=E_{\mathbf{H}}\left\{\operatorname{Pr}\left\{\mathbf{x}_{k} \rightarrow \hat{\mathbf{x}}_{k} \mid \mathbf{H}\right\}\right\} \\
=\frac{1 / 12}{\left(\operatorname{det}\left(\mathbf{I}_{N}+q_{1} \mathbf{K A}\right)\right)}+\frac{1 / 4}{\left(\operatorname{det}\left(\mathbf{I}_{N}+q_{2} \mathbf{K A}\right)\right)},
\end{gathered}
$$

where $\mathbf{A}=\operatorname{diag}\left\{\mathbf{x}_{\mathbf{k}}^{\prime}-\hat{\mathbf{x}}_{\mathrm{k}}^{\prime}\right\}^{H} \operatorname{diag}\left\{\mathbf{x}_{\mathrm{k}}^{\prime}-\hat{\mathbf{x}}_{\mathrm{k}}^{\prime}\right\} ; \mathbf{K}=E\left\{\mathbf{H H}^{H}\right\}$ is the covariance matrix of $\mathbf{H} ; q_{1}=1 /\left(4 N_{0}\right)$ and $q_{2}=$ $1 /\left(3 N_{0}\right)$. After obtaining the unconditional PEP, an upper bound on BER can be derived according to the union bounding technique as [21]

$$
P_{e} \leq \frac{1}{m 2^{m}} \sum_{\mathbf{x}_{k}} \sum_{\hat{\mathbf{x}}_{k} \neq \mathbf{x}_{k}} d\left(\mathbf{x}_{k} \rightarrow \hat{\mathbf{x}}_{k}\right) \operatorname{Pr}\left\{\mathbf{x}_{k} \rightarrow \hat{\mathbf{x}}_{k}\right\},
$$

where $d\left(\mathbf{x}_{k} \rightarrow \hat{\mathbf{x}}_{k}\right)$ measures the number of bits in error between $\mathbf{x}_{k}$ and $\hat{\mathbf{x}}_{k}$.

\section{Simulation Results}

In this section, we perform computer simulations to evaluate the BER performance of JM-OFDM-SNM and AJM-OFDM-

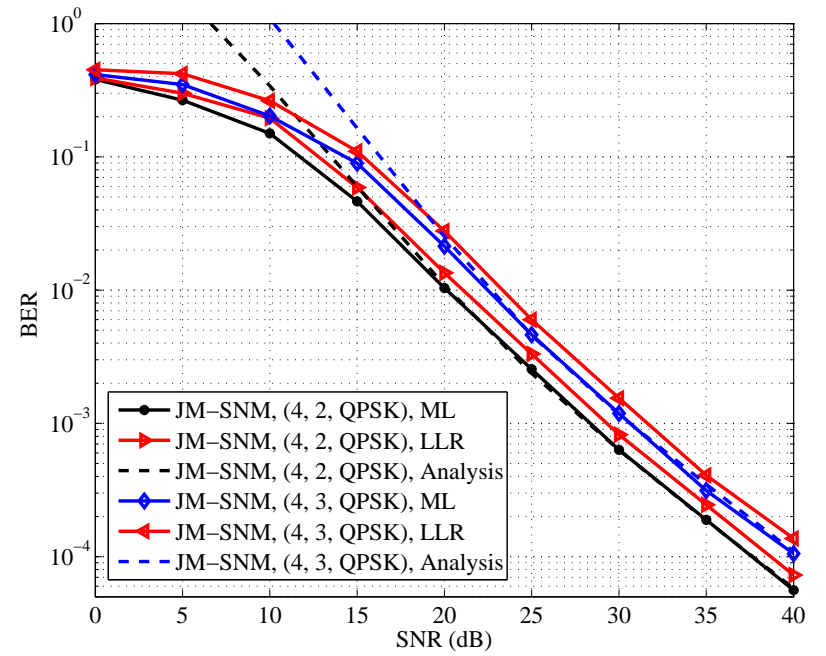

Fig. 2. Performance comparison between ML detection and the proposed low-complexity detection for JM-OFDM-SNM with $N=4$.

SNM schemes under the assumption of Rayleigh fading channels and perfect channel estimation. For convenience, we adopt the shorthands "JM-SNM, ( $N, V, M$ PSK/QAM)" as the JM-OFDM-SNM scheme with $N$ subcarriers, $V$ maximal active subcarriers and the $M$-ary PSK/QAM constellation, and "AJM-SNM, $\left(N, V,\left[M_{1}\right.\right.$ PSK/QAM $, \ldots, M_{V}$ PSK/QAM $\left.]\right)$ " as the AJM-OFDM-SNM scheme with $N$ subcarriers, $V$ maximal active subcarriers, and $V$ constellations, where $M_{v}$ PSK/QAM is allocated to the SAP with $v$ acitve subcarriers $(1 \leq v \leq V)$.

In Fig. 2, we compare the BER performance between the conventional ML detection and the low-complexity detection for "JM-SNM (4, 2, QPSK)" and "JM-SNM (4, 3, QPSK)". It can be seen from Fig. 2 that the proposed LLR detection closely approaches the ML detection with an acceptable performance loss. To be specific, the performance gap between the LLR detection and ML detection is about $1 \mathrm{~dB}$ over the entire SNR region for both "JM-SNM (4, 2, QPSK)" and "JM-SNM (4, 3, QPSK)". Note that the small performance loss stems from the erroneous estimation on the number of active subcarriers $\hat{v}$. Beside, we draw the BER curves yielded by the theoretical analysis for "JM-SNM (4, 2, QPSK)" and "JM-SNM (4, 3, QPSK)". It is found from the figure that theoretical curves of both "JM-SNM (4, 2, QPSK)" and "JMSNM (4, 3, QPSK)" well match the simulation curves in the high SNR region The convergence validates our performance analysis presented in Section III.

To clearly compare the BER performance between the ML detection and the proposed LLR detection, we further carry out the simulations for JM-OFDM-SNM with $N=8$ and present 


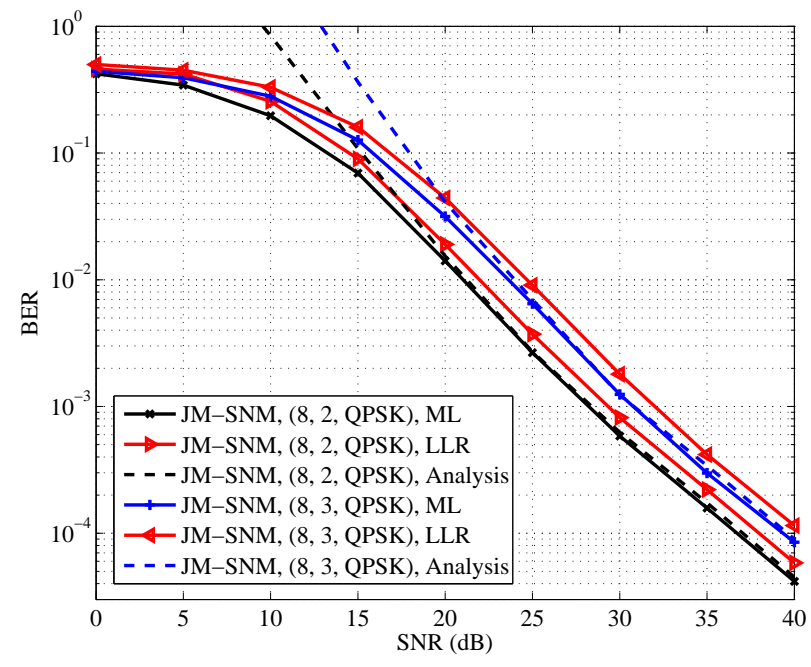

Fig. 3. Performance comparison between ML detection and the proposed low-complexity detection for JM-OFDM-SNM with $N=8$.

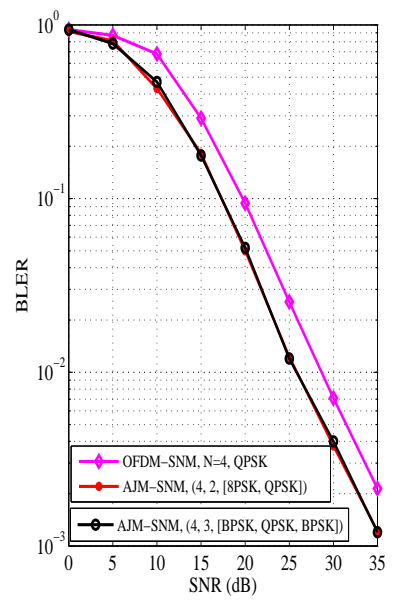

(a)

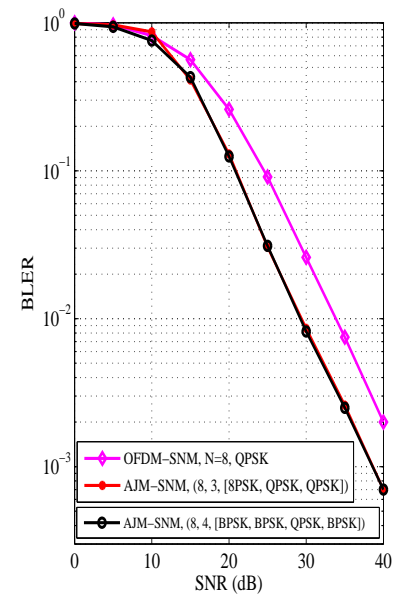

(b)
Fig. 4. Performance comparison between OFDM-SNM and AJM-OFDMSNM. (a) 1.75 bpcu; (b) 1.5 bpcu.

the obtained results in Fig. 3. Specifically, the LLR detection achieves very close performance to the optimal ML detection for "JM-SNM (8, 2, QPSK)" with only a $0.8 \mathrm{~dB}$ performance loss over the entire SNR region. Similarly, the LLR detection also achieves good BER performance, which tightly approaches the ML detection with a constant performance loss of 0.8 $\mathrm{dB}$ for "JM-SNM $(8,3$, QPSK)". We can also find that the theoretical analysis well predicts the simulation results in the high SNR region for "JM-SNM (8, 2, QPSK)" and "JM-SNM (8, 3, QPSK)". Overall, from Figs. 2 and 3, we observe that the proposed LLR detection well approaches the optimal ML detection with a slight performance loss over the entire SNR region, which makes the LLR detection feasible for practical implementations due to its much lower complexity.

In Fig. 4, we compare the block error ratio (BLER) perfor- mance of (A)JM-OFDM-SNM and OFDM-SNM with $N=4$ and $N=8$. Because it is hard to make a fair comparison between JM-OFDM-SNM and OFDM-SNM with the same transmission rate, we alternatively select the AJM-OFDMSNM for comparison with OFDM-SNM. Specifically, we draw the BER curves of "AJM-SNM, (4, 2, [8PSK, QPSK])" $(V=2)$, and OFDM-SNM with $N=4$ and QPSK both under the transmission rate of 1.75 bpcu in Fig. 4(a). It can be seen that "AJM-SNM, (4, 2, [8PSK, QPSK])" obtains a $3 \mathrm{~dB}$ performance gain than OFDM-SNM with $N=4$ and QPSK in the region of SNR $>25 \mathrm{~dB}$. To further exam the performance of AJM-OFDM-SNM with different number of $V$, the BLER performance of "AJM-SNM, (4, 3, [BPSK, QPSK, BPSK])" $(V=3)$ with the transmission rate of $1.75 \mathrm{bpcu}$ is depicted, and we find that "AJM-SNM, (4, 3, [BPSK, QPSK, BPSK])" still outperforms OFDM-SNM with $N=4$ and QPSK with a $3 \mathrm{~dB}$ performance gain. We then compare the BLER performance of "AJM-SNM, (8, 3, [8PSK, QPSK, QPSK])", "AJMSNM, (8, 4, [BPSK, BPSK, QPSK, BPSK])" and OFDM-SNM with $N=8$ and QPSK under the transmission rate of 1.5 bpcu in Fig. 4(a). From Fig. 4(a), we observe that both "AJMSNM, (8, 3, [8PSK, QPSK, QPSK])" and "AJM-SNM, (8, 4, [BPSK, BPSK, QPSK, BPSK])" achieve better performance almost over the entire SNR region with the a constant $4 \mathrm{~dB}$ performance gain for $S N R \geq 20 \mathrm{~dB}$, which further validates the performance advantage of (A)JM-OFDM-SNM. Interestingly, we can see from Fig. 4 that "AJM-SNM, (4, 2, [8PSK, QPSK])" and "AJM-SNM, (8, 3, [8PSK, QPSK, QPSK])" achieve almost the same BLER performance as "AJM-SNM, $(4,3$, [BPSK, QPSK, BPSK])" and "AJM-SNM, (8, 4, [BPSK, BPSK, QPSK, BPSK])" in the high SNR region, respectively. These phenomena can be explained by the fact that the SAP in AJM-OFDM-SNM is likely to be correctly estimated, while the detection error only occurs at the estimation of modulated symbols.

To verify the system performance, we compare the performance of (A)JM-OFDM-SNM with $N=4$ and $V=3$ under the different configurations in Fig. 5. "AJM-SNM, (4, 3 , [BPSK, BPSK, QPSK])" with a transmission rate of $2 \mathrm{bpcu}$ bring out good performance "JM-SNM, (4, 3, QPSK)" with a transmission rate of $2 \mathrm{bpcu}$ in the low SNR region. However, "AJM-SNM, (4, 3, [BPSK, BPSK, QPSK])" and "JM-SNM, $(4,3$, QPSK)" have quite similar performance in the high SNR region because of the dominance of transmitted vector with QPSK and $v=3$. Compared to "AJM-SNM, (4, 3, [BPSK, BPSK, QPSK])" and "JM-SNM, (4, 3, QPSK)", transmitted vectors with 8PSK with $v=2$ are dominant in "AJM-SNM, $(4,3$, [BPSK, 8PSK, QPSK])" with 2.25 bpcu, which results in slightly worse performance. Additionally, "AJM-SNM, (4, 3, [BPSK, QPSK, BPSK])" with a transmission rate of $1.75 \mathrm{bpcu}$ obtains the best performance due to its dominant transmitted vectors with only QPSK and $v=2$. Interestingly, we observe from Fig. 5 that slightly increasing the length of information bits (e.g., 1 more bit) will not affect the BER performance by properly adjusting the cardinality of the constellation set, which is the main advantage of AJM-OFDM-SNM. 


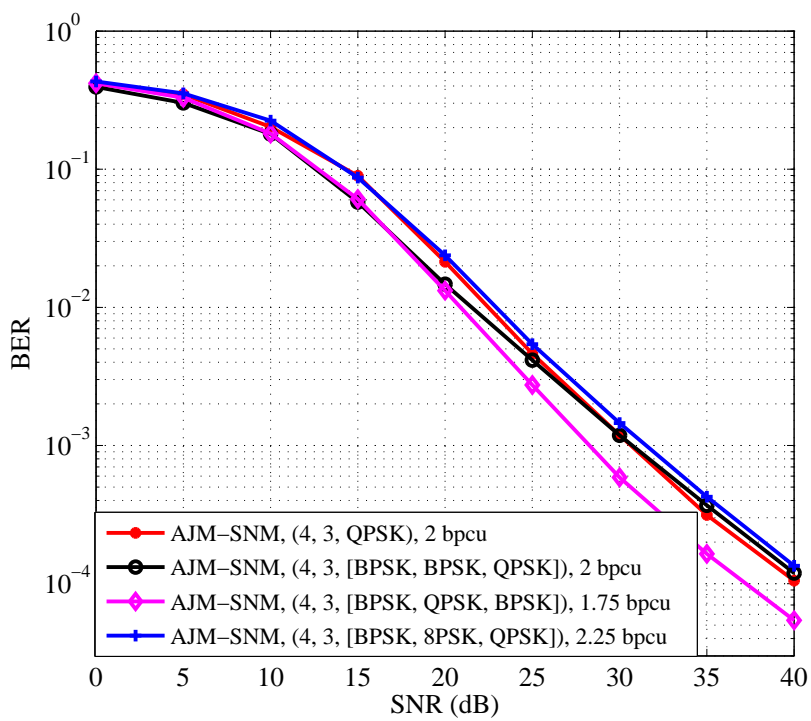

Fig. 5. Performance comparison of AJM-OFDM-SNM with $N=4$ and $V=3$.

\section{CONCLUSIONS}

We proposed the JM-OFDM-SNM scheme to obtain better performance than OFDM-SNM by jointly considering the SAPs and modulation symbols for different numbers of active subcarriers in this paper. To reduce the high detection complexity, we proposed the low-complexity detection for JMOFDM-SNM, which obtains very close performance to ML detection over the entire SNR region. The theoretical analysis was also investigated to evaluate the BER performance of JMOFDM-SNM. To suit different application scenarios, the AJMOFDM-SNM scheme was proposed to adjust the constellation orders for different numbers of active subcarriers so as to improve the SE and the BER performance of JM-OFDMSNM, Computer simulations show that the (A)JM-OFDMSNM scheme outperforms the OFDM-SNM shceme under the same SE. It should be noted that applying the proposed scheme to device-to-device (D2D) systems [22]-[24] will be considered as our future work.

\section{ACKNOWLEDGMENT}

This work was supported by the International Collaborative Research Program of Guangdong Science and Technology Department under Grant No.2020A0505100061, and the National Nature Science Foundation of China (61872102, 61871190).

\section{REFERENCES}

[1] S. Dang, O. Amin, B. Shihada, and M.-S. Alouini, "What should 6G be?," Nature Electronics, vol. 3, pp. 20-29, Jan. 2020.

[2] P. Chen, Z. Xie, Y. Fang, Z. Chen, S. Mumtaz and J. J. P. C. Rodrigues, "Physical-layer network coding: An efficient technique for wireless communications," IEEE Network, vol. 34, no. 2, pp. 270-276, Mar. 2020.
[3] M. S. Omar et al., "Multiobjective optimization in 5G hybrid networks," IEEE Internet Things J., vol. 5, no. 3, pp. 1588-1597, June 2018.

[4] E. Basar, M. Wen, R. Mesleh, M. Di Renzo, Y. Xiao, and H. Haas, "Index modulation techniques for next-generation wireless networks," IEEE Access, vol. 5, pp. 16693-16746, 2017.

[5] M. Wen, B. Zheng, K. J. Kim, M. D. Renzo, T. A. Tsiftsis, K-C. Chen, and N. Al-Dhahir, "A survey on spatial modulation in emerging wireless systems: Research progresses and applications," IEEE J. Sel. Areas Commun., vol. 37, no. 9, pp. 1949-1972, Sept. 2019.

[6] E. Basar, U. Aygolu, E. Panayirci, and H. V. Poor, "Orthogonal frequency division multiplexing with index modulation," IEEE Trans. Signal Process., vol. 61, no. 22, pp. 5536-5549, Nov. 2013.

[7] Y. Xiao, S. Wang, L. Dan, X. Lei, P. Yang, and W. Xiang, "OFDM with interleaved subcarrier-index modulation," IEEE Commun. Lett., vol. 18, no. 8, pp. 1447-1450, Aug. 2014.

[8] M. Wen, B. Ye, E. Basar, Q. Li, and F. Ji, "Enhanced orthogonal frequency division multiplexing with index modulation, "IEEE Trans. Wireless Commun., vol. 16, no. 7, pp. 4786-4801, July 2017.

[9] A. I. Siddiq, "Low complexity OFDM-IM detector by encoding all possible subcarrier activation patterns," IEEE Commun. Lett., vol. 20, no. 3, pp. 446-449, Mar. 2016.

[10] R. Fan, Y. J. Yu, and Y. L. Guan, "Generalization of orthogonal frequency division multiplexing with index modulation," IEEE Trans. Wireless Commun., vol. 14, no. 10, pp. 5350-5359, Oct. 2015.

[11] J. Li, S. Dang, M. Wen, X.-Q. Jiang, Y. Peng, and H. Hai, "Layered orthogonal frequency division multiplexing with index modulation," IEEE Syst. J., vol. 13, no. 4, pp. 3793-3802, Dec. 2019.

[12] E. Basar, "On multiple-input multiple-output OFDM with index modulation for next generation wireless networks," IEEE Trans. Signal Process., vol. 64, no. 15, pp. 3868-3878, Aug. 2016.

[13] T. Mao, Z. Wang, Q. Wang, S. Chen, and L. Hanzo, "Dual-mode index modulation aided OFDM," IEEE Access, vol. 5, pp. 50-60, 2017.

[14] M. Wen, E. Basar, Q. Li, B. Zheng, and M. Zhang, "Multiple-mode orthogonal frequency division multiplexing with index modulation," IEEE Trans. Commun., vol. 65, no. 9, pp. 3892-3906, Sept. 2017.

[15] J. Crawford, E. Chatziantoniou, and Y. Ko, "On the SEP analysis of OFDM index modulation with hybrid low complexity greedy detection and diversity reception," IEEE Trans. Veh. Technol., vol. 66, no. 9, pp. 8103-8118, Sept. 2017.

[16] J. Li, Q. Li, S. Dang, M. Wen, X.-Q. Jiang, and Y. Peng, "Lowcomplexity detection for index modulation multiple access," IEEE Wireless Commun. Lett., vol. 9, no. 7, pp. 943-947, July 2020.

[17] A. M. Jaradat, J. M. Hamamreh, and H. Arslan, "OFDM with subcarrier number modulation," IEEE Wireless Commun. Lett., vol. 7, no. 6, pp. 914-917, Dec. 2018.

[18] S. Dang, G. Ma, B. Shihada, and M.-S. Alouini, "Enhanced orthogonal frequency-division multiplexing with subcarrier number modulation," IEEE Internet Things J., vol. 6, no. 5, pp. 7907C7920, Oct. 2018.

[19] A. M. Jaradat, J. M. Hamamreh, and H. Arslan, "OFDM with hybrid number and index modulation," IEEE Access, vol. 8, pp. 55042-55053, 2020.

[20] M. Chiani and D. Dardari, "Improved exponential bounds and approximation for the Q-function with application to average error probability computation," in Proc. IEEE Global Telecommun. Conf., Bologna, Italy, 2002, pp. 1399-1402.

[21] J. G. Proakis, Digital Communications, 3rd ed. New York: McGraw-Hill, 1995.

[22] S. Mumtaz, K. M. Saidul Huq, J. Rodriguez, and V. Frascolla, "Energeefficient interference management in LTE-D2D communication," IET Signal Process., vol. 10, no. 3, pp. 197-202, 2016.

[23] M. Neyja, S. Mumtaz, K. M. S. Huq, S. A. Busari, J. Rodriguez and Z. Zhou, "An IoT-based E-health monitoring system using ECG signal," in Proc. IEEE Global Commun. Conf., Singapore, 2017, pp. 1-6.

[24] S. Khan, K. Muhammad, S. Mumtaz, S. W. Baik and V. H. C. de Albuquerque, "Energy-efficient deep CNN for smoke detection in foggy IoT environment," IEEE Internet Things J., vol. 6, no. 6, pp. 9237-9245, Dec. 2019. 